\title{
Impact of hedonic evaluation on the expected consumers' preferences for beef attributes including its enrichment with $n-3$ and CLA fatty acids
}

\author{
Yasmina Baba ${ }^{1}$, Zein Kallas ${ }^{2 *}$, Carolina E. Realini ${ }^{3}$, José Maria Gil ${ }^{2}$, Montserrat Costa-Font ${ }^{2}$ \\ ${ }^{1}$ Instituto de Sostenibilidad, Universidad Politécnica de Cataluña, Plaza Eusebi Güell, 6. 08034 Barcelona, Spain. \\ ${ }^{2}$ Centre for Agro-food Economy and Development (CREDA), Esteve Terradas, 8, 08860 Castelldefels, Spain. \\ ${ }^{3}$ IRTA-Monells, Finca Camps I Armet, 17121 Monells, Spain.
}

\author{
${ }^{*}$ Corresponding author. \\ CREDA-UPC-IRTA \\ Parc Mediterrani de la Tecnologia \\ Edifici ESAB \\ Esteve terrades, 8 \\ 08860 \\ Castelldefels -(Barcelona), Spain \\ Phone: + (34) 935521213 \\ Fax: + (34) 935521121
}

E-mail address: zein.kallas@upc.edu (Zein Kallas) 


IMPACT OF HEDONIC EVALUATION ON THE EXPECTED
CONSUMERS' PREFERENCES FOR BEEF ATTRIBUTES INCLUDING
ITS ENRICHMENT WITH $n$-3 AND CLA FATTY ACIDS

\begin{abstract}
The impact of hedonic evaluation on the expected consumers' preferences towards beef attributes including its enrichment with polyunsaturated fatty acids (PFA) was evaluated. Six hundred and forty seven Spanish consumers were divided into two groups differentiated by the information received. Consumers assessed five beef attributes (origin, animal diet, amount of visible fat, meat colour and price) by conducting a discrete choice experiments (DCE) using the Generalized Multinomial Logit model (G-MNL). Subsequently, after a blind tasting of beef samples, consumers repeated the DCE. Results showed that the hedonic evaluation had a significant impact on consumer beef preferences, in particular, for the animal diet attribute. After tasting, the scale heterogeneity, which is the variation of the degree of randomness in the decision-making process and hence the degree of individuals' certainty, have decreased significantly. Results showed higher consumers' overall acceptability scores for beef enriched with PFA, and the information offered to consumers had no significant impact on their acceptability scores.
\end{abstract}

Key Words: n-3, CLA, beef meat, choice experiments, Generalized Multinomial Logit model. 


\section{Introduction}

When health claims are presented on food package, purchase intentions are favourably influenced and consumers risks perception of certain diseases decrease (Kozup, et al., 2003). Consumers are more aware of the contribution of food to their health (Siró et al. 2008) and thus, health concerns are becoming a main determinant factor for food consumption. To meet today's health and wellness concerns, food/beverage demands has evolved towards new range of products often related to health-promotion and disease prevention. In United States, health influenced the food purchase decisions of $64 \%$ of consumers in 2013 , up from $61 \%$ in 2012 (IFIC, 2013). However, consumer perception and purchase behaviour of functional ingredients is not one-dimensional, and the final food result from a variety of factors such as sensory, socioeconomic, attitudinal, risk perception, cultural and information issues among others (Hellyer et al, 2012; Siró et al., 2008; Urala and Lähteenmäki, 2004).

Cultural and attitudinal factors plays an important role in food choice. Siró et al. (2008) stated that there is a clear difference between western and eastern valuation of functional food. Western perception of maintenance of original food characteristics is more important for Europeans than North Americans. The Mediterranean consumers are the ones more concerned with the "natural" characteristic of food. Therefore, the balance between the valuation of health effects/benefits of specific functional ingredients and the preservation of the original food characteristics are key points for the acceptability of functional food. In this context, Franchi (2012) mentioned that beliefs and identity are influence preferences by indicating to individuals what foods are 'good' and 'right'.

Sensory attributes are also decisive factors for acceptance of food especially those dealing with health claims and well-being enhancement (Verbeke, 2005; Urala and Lähteenmäki, 2003 \& 2004). Gabrielyan, et al. (2014) mentioned that the intrinsic cues such as taste are a primary basis for consumers' expectations of quality and decisions about whether to make repeat purchases of a product. Asioli et al. (2014) found that flavour and odour are the most important in driving consumers' choice for organic food. Annett et al. (2008) and Hobbs et al. (2006) verified that health and nutrition information together with sensory evaluation and eating experience are all relevant for a positive valuation of specific functional food (organic bread/functional meat). Combris et al. (2009) noticed that personal experience, derived from a blind tasting, was significantly more important than label information regarding "appellation of 
origin" of wines. That is, experience plays a very important role in defining individuals' perception and willingness to pay. Lange et al. (2002) and Noussair et al., (2004) compared hedonic ratings and experimental auctions to evaluate food preferences, stating that hedonic ratings provided similar aggregate results. Poole et al. (2006) employed an experimental auction to test fruit quality perceptions by evaluating consumers' willingness to pay (WTP) after three alternative sensory experiments (visual appearance, touching and peeling, and tasting). The authors concluded that "experience" modifies product quality perceptions and scoring behaviour, as well as it is likely to affect repurchase decisions. Lange et al. (1998) compared consumers' behaviour using two scenarios: just packaging exposure and packaging exposure and taste. The authors reported that tasting had an important role on consumers' purchase decisions. Respondents do consider different food attributes after tasting than before tasting with a modification on their purchase decisions.

Many studies have analysed consumers' preferences, attitudes and acceptance towards beef (Carpenter et al., 2001; Resurreccion, 2004; Verbeke et al., 2010; Font-i-Furnols \& Guerrero, 2014 among others). However, literature that analyses the impact of hedonic valuation on consumers' purchasing decisions toward new developed meat products is still scarce and remains untreated for beef enriched with polyunsaturated fatty acids, particularly in Spain. In this context, we applied a methodological approach that attempts to mimic consumers' behaviour towards a novel product (enriched beef with beneficial fatty acids), which can be summarized in 3 main subsequent steps:

a) When consumers face a new product on the shelf stores, they generate expectations (expected or pre-sensory preferences) on the basis of their past experiences and available information related to the characteristics of the product or to similar products (Deliza \& MacFie, 1996).

b) Tasting the new product (hedonic evaluation test) allow constructing a set of current experience information that is useful to decide for a repeated choice or not.

c) After tasting the new product, consumers' acceptance may result in agreement or disagreement with what they expected. These changes play an important role in the acceptance or rejection of the new product (Font-i-Furnols \& Guerrero, 2014), and may affect the final choice of the consumers (final or post-sensory preferences).

In this context, the main objective of this paper was to analyse the impact of hedonic evaluation, for both informed and non-informed groups of consumers, on the expected preferences for beef 
100 attributes including its enrichment with polyunsaturated fatty acids (omega-3 and the conjugated

101 linoleic acid, CLA). The analysis of the hedonic evaluation impact is carried out by comparing the 102 consumers' preferences before and after the tasting experience. In addition, we analysed if the 103 health information delivered to consumers had influenced the overall acceptability scores for 104 beef. From one hand, empirically, this is the first paper that analysed the sensory impact on the 105 expected preferences towards the enriched beef meat with polyunsaturated fatty acid. On the 106 other hand, methodologically, this paper contribute to the literature of the Discrete Choice 107 Modelling (DCM) using the recently developed Generalised Multinomial Logit Model (G-MNL) of 108 Fiebig et al., (2010) allowing for both preference and scale heterogeneity. To our knowledge, 109 this is the first application, in the literature of food and meat preferences studies that analyse the 110 impact of sensory experience on consumers' preferences using the G-MNL and that analyses 111 how the scale heterogeneity is affected.

112

2. Materials and methods

114 In accordance to the main objective, our methodological framework consisted of three main 115 steps:

116 a) The first part focussed on analysing the expected consumers' preferences using discrete 117 choice experiments (DCE) towards beef meat attributes and its enrichment with n-3 and 118 CLA (expected or pre-sensory preferences step). In this initial step, consumers were divided 119 into two groups. While the first one received information about the enrichment process and 120 the health benefits of CLA and n-3 fatty acids, the second group did not receive any 121 additional information.

122 b) The second part was based on a blind tasting of four types of beef samples (conventional, 123 enriched with n-3, enriched with CLA and enriched with both n-3 and CLA) from animals fed one of four different diets (hedonic evaluation test step). In this second stage, consumers' overall acceptability was assessed using a 9-point hedonic scale ( 1 = dislike extremely to 9 $=$ like extremely). After tasting of samples, all consumers were told what type of beef they have tasted in order to associate their score with the different types of beef meat.

132 A summarized scheme of the methodological framework is presented in Figure 1. As can be 133 seen, this approach allowed first to analyse the impact of health information on the expected 
134 preferences (point 1) that has been reported by Kallas et al. (2014). It also permitted analysing 135 the hedonic evaluation regarding beef attributes in particular the n-3 and CLA attributes (point 2)

136 that has been presented and discussed by Realini et al. (2014). Finally, the hedonic evaluation

137 impact on the expected preferences of informed and non-informed consumers is assessed in

138 this study.

\subsection{Theoretical foundation of the Discrete Choice Experiments}

141 The preference analysis is based on the DCE that aims to identify the individual's indirect utility 142 function associated with attributes of products by examining the trade-offs they make when 143 making choice decisions. Thus, several alternatives that are described by several attributes with 144 varying levels are presented to respondents in choice sets. The respondent is then asked to select 145 its preferred alternative within each choice set, thereby revealing his/her preference for certain 146 attributes and levels. Subsequently, the relative importance of the attributes can be indirectly 147 recovered from respondents' choices.

148 DCE rely on Lancaster's Theory of Value (Lancaster, 1966) which proposes that utility of a product 149 is decomposed into separable utilities for their characteristics or attributes. It is also based on the 150 Random Utility Theory (RUT) laid out by Thurstone (1927). This theory propose that subjects 151 choose among alternatives according to a utility function with two main components: a systematic 152 (observable) component and a random error term (non-observable):

$$
U_{j n}=V_{j n}\left(X_{j}, S_{n}\right)+\varepsilon_{j n}
$$

154 where $U_{j n}$ is the utility of alternative $j$ to subject $n, V_{j n}$ is the systematic component of the utility,

$155 X_{j}$ is the vector of attributes of alternative $j, S_{n}$ is the vector of socio-economic characteristics of 156 the subject $n$ and $\varepsilon_{j n}$ is the random term.

\subsection{Choice Experiments modelling}

159 To predict the subjects' preferences for attributes (k), we need to define the "probability of choice" 160 that an individual $n$ chooses the alternative $i$ rather than the alternative $j$ (for any $i$ and $j$ within 161 choice sets, $T$ ). McFadden (1974) developed an econometric model that formalized respondents' 162 decision making process. This model is often referred to as the multinomial logit (MNL) model, 163 which is considered the base model for DCE. According to MNL model the utility to person $n$ from 164 choosing alternative $j$ on choice scenario $t$ is given by: 
$U_{n j t}=\beta x_{n j t}+\varepsilon_{n j t} / \sigma_{n} \quad n=1, \ldots, N \quad j=1, \ldots, J \quad t=1, \ldots, T$

166 Where, $x_{n j t}$ is a vector of observed attributes of alternative $j, \beta$ is a vector of mean attribute

167 utilities (utility weights) and $\varepsilon_{n j t}$ is the "idiosyncratic" error term that follows independent and

168 identically distributed (i.i.d.) Type 1 extreme value distribution with scale parameter $\sigma_{n}$.

170 The probability $\left(P_{j} \mid X_{n t}\right)$ that an individual $n$ will choose alternative $j$ among other alternative of 171 an array of choice set $\mathrm{T}$ is formulated as follows:

172

$$
\left(P_{j} \mid X_{n t}\right)=\frac{\exp \left(\beta x_{n j t}\right)}{\sum_{j=1}^{J} \exp \left(\beta x_{n j t}\right)} \quad \forall j \in T
$$

174 Where $X_{n t}$ is the vector of attributes of all alternatives $j=1, \ldots, J$. In the case of estimating a

$175 \mathrm{MNL}$, the scale parameter $\sigma_{n}$ is normalized to one for identification.

176 The MNL impose homogeneity in preferences for observed attribute. Thus, only average attributes' 177 utilities are estimated which is often unrealistic as consumers' preferences are, by nature, 178 heterogeneous. Therefore, the mixed or heterogeneous logit models (MIXL) (in the literature is 179 also referred to as Random Parameter Logit model, RPL) have been introduced to investigate 180 such heterogeneity. This model extend the MNL allowing for unobserved heterogeneity by 181 allowing random coefficients on attributes (Ben-Akiva et al., 1997).

182 In MIXL the utility to person $n$ from choosing alternative $j$ in choice set $t$ is given by:

$183 U_{n j t}=\beta_{n} x_{n j t}+\varepsilon_{n j t} / \sigma_{n} \quad n=1, \ldots, N j=1, \ldots, J \quad t=1, \ldots, T$

184 Where, $\beta_{n}=\beta+\eta_{n}$ and where $\left(\eta_{n}\right)$ is the vector of person $n$ specific deviations from the mean 185 value of the $\beta \mathrm{s}$. The $\eta_{n}$ is described by an underlying continuous distribution for the attributes 186 defined by the researcher. In most applications the multivariate normal distribution is the most 187 used, $\operatorname{MVN}(0, \Sigma)$. In this case, $\sigma_{n}$ is also assumed to be one for identification. This model has 188 been used in the analysis regarding the information impact on expected preferences (point 1 in 189 Figure 1). However, Louviere and Mayer (2007) and Louviere et al. (2008) argued that much of 190 the preference heterogeneity captured by random parameters can be better captured by the scale 191 term; and thus known as "scale heterogeneity". Therefore, they considered that the MIXL turns to 
192 be likely a poor approximation to stated data if scale heterogeneity is not accounted for (Fiebig, et 193 al., 2010).

194 The scale heterogeneity is the variation of the degree of randomness in the decision-making 195 process over respondents and hence is the degree of individuals' certainty. It is based the 196 differences of the variance of the error term $(\varepsilon)$ across individual-decision-makers. In this context, 197 the analysis of the scale heterogeneity is important, especially for the stated preference studies 198 (i.e. based on questionnaire). In this context, Feibig et al. (2010) developed the Generalized 199 Multinomial Logit model (GMNL). Within this approach, the $\sigma_{n}$ is no longer set to be one, and a 200 particular specification of this term is assumed. Feibig et al. (2010) identified that the utility to 201 person $n$ from choosing alternative $j$ on choice set $t$ is given by:

202

$U_{n j t}=\left[\sigma_{n} \beta+\gamma \eta_{n}+(1-\gamma) \sigma_{n} \eta_{n}\right] X_{n j t}+\varepsilon_{n j t}$

203 where $\gamma$ is a parameter between 0 and 1 . It is a mixing parameter, and its value determines the 204 level of mixing or interaction between the scale heterogeneity coefficient $\sigma_{n}$ and the parameter 205 heterogeneity coefficient $\eta_{n} . \sigma_{n}$ is a scaling factor that proportionately scales the $\beta$ up or down 206 for each individual $n$. Finally, because $\sigma_{n}$ only enters the model as a product of $\sigma_{n} \beta$ (equation 5), 207 Fiebig et al. (2010) proposed $\sigma_{n}=\exp \left(\bar{\sigma}+\tau v_{n}\right)$ and $\tau$ is estimated ${ }^{1}$.

208 The GMNL model is specified by default to consider the $\eta_{n}$ as uncorrelated. That is mean the 209 covariance matrix of $\eta_{n}$ is constrained to be a diagonal matrix (a matrix in which all values above 210 and to the right of the diagonal are equal to zero). However, the GMNL can be specified to allow 211 for correlated parameters. The presence of multiple observations on stated-choice responses for 212 each sampled individual means that the potential for correlated responses across observations 213 can be the product of many sources including the sequencing of offered choice situations that 214 results in mixtures of learning and inertia effects, among other possible influences on choice 215 response as commented by Hensher et al., (2005). Thus, discrete choice data with a repeated 216 choice situations containing the same attributes and levels may have unobserved effects that are 217 correlated among alternatives in a given choice situation. When the random parameter are 218 correlated the model reports the diagonal value of the Cholesky matrix that represent the true 219 standard deviation for each random parameter once the cross-correlated parameter terms have 220 been unconfounded. The below -diagonal elements in Cholesky decomposition matrix are the

\footnotetext{
${ }^{1}$ More details about the GMNL model can be found in Fiebig et al., (2010) and Louvier et al. (2008).
} 
covariances (cross-correlation) among the random parameter estimates. In this study, we estimated a correlated random parameter within the G-MNL model due to its best goodness of fit. For the estimation, we used the GMXLOGIT procedure in NLOGIT 5 with the "correlated" option.

Once the model is estimated, we calculate the relative importance $\left(I_{k}\right)$ of each attribute. Thus, the ratio of a particular attribute utility to the sum of all attributes' utilities is used to reveal its relative importance by the following equation (Smith, 2005):

$$
I_{k}=\frac{\left(\max \beta_{k}-\min \beta_{k}\right)}{\sum_{k=1}^{K}\left(\max \beta_{k}-\min \beta_{k}\right)}
$$

where $\left(I_{k}\right)$ is the relative importance of the attribute $(k),\left(\max \beta_{k}\right)$ is the maximum utility of the

230 attribute (i.e. the most preferred level) and $\left(\min \beta_{k}\right)$ is the minimum utility (i.e. the least preferred

231 level).

\subsection{Empirical application}

234 The first step in the application of the DCE is the identification of the attributes that best describe 235 the product of interest. For this study, on the basis of the literature review presented in Kallas et 236 al., (2014) and Realini et al., (2014) we defined five attributes: the animal diet with four levels were 237 evaluated which corresponded to the type of beef assessed in the hedonic evaluation 238 (conventional, enriched with omega-3, enriched with CLA and enriched with omega-3 plus CLA).

239 Origin attribute with two levels as 'locally produced' and 'other Spanish origin'. Two meat colour 240 levels were evaluated as 'pale red' or 'bright red', and two fat levels of beef steaks were 241 considered as 'moderate visible fat' or 'slight visible fat'. Finally, beef price was included as 242 another key attribute with four levels: $6.6 €$ high, $5.7 €$ medium-high, $4.8 €$ medium-low and $3.9 €$ 243 low meat price. The second step is to carry out an experimental design that allow for creating the 244 different situation of choice (choice sets). We followed the Dual Response Choice Experiments 245 (DRCE) design (Kallas et al., 2012). An orthogonal fractional factorial design was used, obtaining 24616 choice sets. Finally, factorial blocking arrangement was carried out obtaining 2 blocks, each 247 with 8 choice sets presented to individual respondents so that the number of profiles would be low 248 enough to be easily handled by consumers.

249 The data were obtained from a sample consisted of two different consumer groups. The first 250 sample consisted of 322 consumers that did not receive any information about the enriched meat 251 presented in the choice sets. The second group comprised 325 consumers who received 
252 extensive information about the enrichment process of the beef meat and the advantages of this 253 product to human health. Regarding the beef sampling procedure for hedonic evaluation, the meat 254 sample preparation for consumer liking assessment were obtained from forty-eight Holstein entire 255 males fed with one of four dietary treatments. All animal diets had similar composition but differed 256 in the content of whole linseed and conjugated linoleic acid (CLA): CONV (conventional 257 commercial ration, 0\% linseed and 0\% CLA), OME3 (conventional ration enriched with omega-3 258 fatty acids through the addition of $10 \%$ linseed), CLA (conventional ration enriched with CLA 259 through the addition of $2 \%$ CLA), and OME3-CLA (conventional ration enriched with omega-3 and 260 CLA fatty acids through the addition of $10 \%$ linseed plus $2 \%$ CLA) ${ }^{2}$.

\section{Results and discussion}

\subsection{Impact of hedonic evaluation on expected preferences}

265 Focusing on how sensory experience from the hedonic evaluation affect the expected preferences generated for both informed and non-informed consumers, results of the full GMNL model showed the relative importance of the attributes (Table 1). Regarding the pre sensory test results, the order of the relative importance of the attributes was slightly different for both groups of consumers, showing that the information provided had an impact on their expected beef meat preferences as reported by Kallas et al. (2014). Thus, for non-informed consumers, fat content was the most important attribute while it was less important for the informed ones. There is a clear substitution effect between the diet and the fat content showing the significant impact of information on consumers' preferences. It is evident that consumers are less concerned about the amount of visible fat in beef as long as it is enriched with beneficial fatty acids.

276 In this context, diet was not important in the beef purchasing decisions of uninformed consumers 277 before tasting the product, but it was one of the most important factors for informed consumers 278 and after the hedonic evaluation. These results show that producing enriched beef meat may 279 lead consumers to give less importance to its fat content, assuming that the beneficial 280 compounds (omega-3 and CLA) may counteract the negative effect of the amount of fat. The 281 improvement of the fatty acid composition of beef through modifications in the animal diet would 282 provide consumers with a product that is closer to current nutritional recommendations for a 283 healthy diet, favouring consumers' purchasing decisions regarding enriched meat. In addition,

\footnotetext{
${ }^{2}$ More details about the attributes' selection, the experimental design, the procedure of sample selection and the meat sample preparation can be found in Kallas et al. (2014) and Realini et al., (2014).
} 
consumers would be less concerned about the amount of fat present in enriched meat, which is also positively related with the sensory properties of meat.

Analysing preferences before and after the sensory test within each group, results show significant modifications in the relative importance of the attributes for the non-informed consumers, while minor changes occurred for the informed ones in their beef purchasing preferences. Results showed that after beef tasting, a significant change resulted in the diet preference which has moved from a non-significant preference to the most important one. In addition, the relative importance of the fat attribute decreased significantly.

Results from Table 2 reports the marginal utilities of the attributes, the attribute heterogeneity terms and the scale parameters ${ }^{3}$. As commented, behaviourally, the advantage of the G-MNL is that it allows respondents to have different utility function scales that describe a different uncertainty levels with respect to the choices they make. Focusing on the scale parameters, the information and the hedonic evaluation have had a significant impact. Results shows that moving from models for non-informed consumers to informed one, the average error scales decreased significantly. This may indicate that when the consumers are informed they make more reliable choices. In addition, for the informed consumers both the $\gamma$ and the $\tau$ parameter were not significantly different from zero. Thus the unobserved heterogeneity in this case is better described by the normally distributed deviations from mean coefficients, but there is no additional value in describing it with a scaling factor. It is also relevant that the hedonic evaluation for both informed and non-informed consumers showed some evidence of a shift in the scaling factor across choice ( $\tau$ turns to be insignificant) showing that consumers after tasting experience tended to be more reliable about their choice exhibiting non-significant scale heterogeneity.

Focusing on each attributes levels, results show those preferences for informed and non-informed consumers before and after tasting beef samples from animals fed different diets intended to

312 improve the fatty acid profile of meat. To better understand the attribute preferences, the utilities of

313 the different levels of each attribute from the GMNL estimation were obtained. Utilities for the 314 amount of visible fat were higher for the uninformed consumers, which indicates that consumers 315 that do not receive information about the benefits of omega-3 and CLA fatty acids or their role in 316 human health are more concerned about the amount of fat in meat. There is a reduction in the

\footnotetext{
${ }^{3}$ Results of the estimated covariances of the attributes from the Cholesky matrix (45 parameters) are available upon request.
} 
317 utilities for the fat attribute after tasting for non-informed consumers since the relative importance

318 of this attribute decreases. After the blind tasting of the different beef samples, consumers were 319 told about the type of beef that they have tasted. Thus, for the non-informed consumers the 320 relative importance of other attributes and their utilities such as the animal diet increased 321 significantly compared with the fat content which decreased. Enriched beef had similar or slightly 322 higher hedonic scores compared with conventional beef (Table 2). Many authors indicated that 323 consumers are not willing to compromise on taste of functional foods for eventual health benefits 324 (Augustin, 2001; Cox, Koster \& Russell, 2004; Gilbert, 2000; Verbeke, 2006). Results from this 325 study indicate that consumers may be less concerned about the amount of fat in meat, if they 326 become aware that sensory properties are not compromised when meat is enriched with beneficial 327 fatty acids.

329 Regarding the levels of meat colour and origin, results show a convergence of preferences for 330 both types of consumers. Moreover, the sensory test had no impact on consumer preferences 331 maintaining the order of the preferred colour (bright red) and the preferred origin (locally produced). 332 For the diet attribute, the utility for the enriched meat with omega-3 increased and the preference 333 for the conventional one decreased for both groups of consumers, but especially for non-informed 334 consumers. However, there is a consensus to reject the CLA enriched beef for expected 335 preference before tasting and that obtained after the sensory analysis. Consumer preferences 336 regarding the diet attribute may be explained by the fact that most consumers are familiar with 337 omega-3 fatty acids and with some commercial products enriched with these fatty acids, in 338 contrast to CLA and the enrichment of food products with CLA. Siró et al. (2008) also indicated 339 that well-known compounds are more accepted than less-known components in food products.

341 Finally, after the tasting experience, there is a reduction of the utility associated with the higher 342 and average prices and a slight increase with the lower price for informed consumers. Focusing 343 on the lower price level, informed consumers showed the highest utility increase. This may 344 indicate that the sensory experience of the enriched meat was not enough to justify per se that the 345 price of the meat have to be more expensive as a results of the enrichment. Regarding the opt-out 346 option, results show that the utility associated with it was not significant for informed consumers, 347 showing that more consumers did not select the opt out option in comparison to the non-informed 348 one. In this later case, the utility of the opt-out was positive and significant which is an indicator 349 that without information some products do not convince consumers, mainly the enriched with CLA 350 and those with high prices. 


\subsection{Impact of information on consumers' beef acceptability}

352 Overall acceptability scores for beef from animals fed the different diets is shown in Table 3.

353 Comparing the overall acceptability of the four types of beef meat, results show a non-significant 354 impact of the information at $95 \%$. This indicates that the information provided to one group of 355 consumers about the benefits of omega- 3 and CLA fatty acids did not have an influence on their 356 hedonic preferences for beef. In contrasts to our results, Morales et al. (2013) showed that 357 information about beef production systems generated positive expectations and increased 358 acceptability ratings for beef from grazing animals. This may indicate that the impact of 359 information on hedonic preferences by consumers may depend on the type of information 360 provided. Since there were no differences in our study in hedonic scores depending on 361 information, we will focus on the values obtained for the whole sample (all consumers) for the 362 interpretation of the sensory scores. Results showed that enriched beef with omega-3 fatty acids 363 had higher acceptability scores than beef from the other treatments. Beef enriched with CLA had 364 similar acceptability scores to beef enriched with both omega-3 and CLA which in turn was 365 similar to conventional beef. It should be noted, however, that the differences among 366 acceptability scores are within 0.5 in a 9 point scale using a high number of consumers $(n=642)$. 367 Results indicate that differences in beef acceptability among dietary treatments, although 368 statistically significant, are not large.

\section{Conclusion}

Results showed that hedonic evaluation had a significant impact on defining consumer beef preferences, especially for non-informed consumers, and thus, their expected preferences were affected by the sensory evaluation. Focusing on the animal diet attribute, utilities for $n-3$ enriched beef increased significantly after tasting, particularly for non-informed consumers, while utilities for CLA enriched beef were still not significant after tasting for all consumers. In this context, after the hedonic evaluation, there was a positive disagreement between the expected preference for $n-3$ enriched meat and the final preference. Provided information about the enrichment process and the health benefits of $n-3$ and CLA fatty acids had no significant impact on overall acceptability scores of beef.

After the hedonic valuation results showed that the unobserved heterogeneity is better described by the normally distributed deviations from mean coefficients and there is no additional value in 383 describing it with a scaling factor. Thus, the beef tasting exhibit for both informed and non384 informed consumers evidence of a shift in the scaling factor across choice. Comparing 
preferences before and after beef tasting results also shows significant changes in the relative importance of some attributes. In this sense, the heterogeneous scale identified before the sensory test tended to be more homogeneous after tasting. The GMN-L model was first estimated with uncorrelated coefficients. Compared to this, the correlated version provide a better fit to the data. Beside preference heterogeneity, we also find statistically significant scale heterogeneity; therefore the assumption of identical scales across individuals is rejected. Results also showed that the full version of the GMN-L (including correlation between random parameter and between taste and scale preferences) had the best goodness of fit (AIC, Pseudo $R^{2}$ ). Analysing the attributes non-attendance before and after sensory are proposed for further research.

\section{ACKNOWLEDGEMENTS}

This research was supported by the National Institute of Agrifood Research (project RTA2009-00004-CO2).

\section{REFERENCES}

Annett, L.E., Muralidharan, V., Boxall, P.C., Cash, S.B. \& Wismer, W.V. (2008). Influence of health and environmental information on hedonic evaluation of organic and conventional bread. Journal of food science 73 (4), 50-57.

Asioli, D., Canavari, M., Pignatti, E., Obermowe, T., Sidali, K. L., Vogt, C., \& Spiller, A. (2014). Sensory experiences and expectations of Italian and German organic consumers. Journal of International Food \& Agribusiness Marketing,26(1), 13-27.

Augustin, M. A. (2001). Functional foods: an adventure in food formulation. Food Australia, 53 (10), 428-432.

Ben-Akiva, M., D. McFadden, M. Abe, U. Böckenholt, D. Bolduc, D. Gopinath, T. Morikawa et al. 1997. Modelling methods for discrete choice analysis. Marketing Lett. 8(3) 273-286

Carpenter, C. E., Cornforth, D. P., \& Whittier, D. (2001). Consumer preferences for beef color and packaging did not affect eating satisfaction. Meat Science, 57(4), 359-363.

Combris, P., Bazoche, P., Giraud-Héraud, E., \& Issanchou, S. (2009). Food choices: What do we learn from combining sensory and economic experiments? Food Quality and Preference, 20(8), 550-557.

Cox, D. N., Koster, A. \& Russell, C. G. (2004). Predicting intentions to consume functional foods and supplements to offset memory loss using an adaptation of protection motivation theory. Appetite, 43 (1), 55-64.

Deliza, R. \& MacFie, H.J. (1996). The generation of sensory expectation by external cues and its effect on sensory perception and hedonic ratings: a review. Journal of Sensory Studies, 11(2), 103-128.

Fiebig, D. G., Keane, M. P., Louviere, J., \& Wasi, N. (2010). The generalized multinomial logit model: accounting for scale and coefficient heterogeneity.Marketing Science, 29(3), 393-421.

Font-i-Furnols, M. \& Guerrero, L. (2014). Consumer preference, behaviour and perception about meat and meat products: an overview. Meat Science.

Franchi, M. (2012). Food choice: beyond the chemical content. International Journal of Food Sciences and Nutrition, 63(S1), 17-28.

Gabrielyan, G., McCluskey, J. J., Marsh, T. L., \& Ross, C. F. (2014). Willingness to Pay for Sensory Attributes in Beer. Agricultural and Resource Economics Review, 43(1), 125-139. 
Gilbert, L. C. (2000). The functional food trend: What's next and what Americans think about eggs. Journal of the American College of Nutrition, 19 (5), 507S-512S.

Hellyer, N., Fraser, I., Haddock-Fraser, J., (2012) Food choice, health information and functional ingredients: An experimental auction employing bread. Food Policy, 37(3), 232-245.

Hensher, D., Rose, J. \& Greene, W. (2005) Applied choice analysis: A primer. Cambridge University Press: Cambridge.

Hobbs, J. E., Sanderson, K. and Haghiri, M. (2006), Evaluating Willingness-to-Pay for Bison Attributes: An Experimental Auction Approach. Canadian Journal of Agricultural Economics/Revue canadienne d'agroeconomie, 54, 269-287.

IFIC. 2013. Food \& health survey. International Food Information Council Foundation, Washington, D.C., available at www.foodinsights.com.

Kallas, Z. \& Gil, J.M. (2012). A dual response choice experiments (DRCE) design to assess rabbit meat preference in Catalonia A heteroscedastic extreme-value model. British Food Journal, 114 (10-11), 1394-1413.

Kallas, Z., Realini, C.E. \& Gil, J.M. (2014) Health information impact on the relative importance of beef attributes including its enrichment with polyunsaturated fatty acids (omega-3 and conjugated linoleic acid). Meat Science. 97(4), 497-503.

Keane, M. (2006). The generalized logit model: Preliminary ideas on a research program. Presentation, Motorola-CenSoC Hong Kong Meeting, October 22, Motorola, Hung Hom, Kowloon, Hong Kong.

Kozup, J. C., Creyer, E. H., \& Burton, S. (2003). Making healthful food choices: The influence of health claims and nutrition information on consumers' evaluations of packaged food products and restaurant menu items. Journal of Marketing, 67(2), 19-34.

Lancaster, K. (1966) A new approach to consumer theory. Journal of Political Economy, 74, 132 57.

Lange, C., Martin, C., Chabanet C., Combris, P. \& Issanchou, S. (2002). Impact of the information provided to consumers on their willingness to pay for Champagne: Comparison with hedonic scores. Food Quality and Preference, 597-608.

Lange, C., Rousseau, F. \& Issanchou, S. (1998). Expectation, liking and purchase behaviour under economical constraint, Food Quality and Preference, 10(1), 31-39

Louviere, J. J. \& Meyer, R. J. (2007). Formal choice models of informal choices: What choice modeling research can (and can't) learn from behavioral theory. N. K. Malhotra, ed. Review of Marketing Research. M. E. Sharpe, New York, 3-32.

Louviere, J. J., Street, D. Burgess, L., Wasi, N. Islam, T. \& Marley, A.A. (2008). Modelling the choices of individuals' decision makers by combining efficient choice experiment designs with extra preference information. Journal of Choice Model, 1(1) 128-163.

16-29.

McFadden, D. (1974) Conditional logit analysis of qualitative choice behavior. In P. Zarembka, (Ed) Frontiers in econometrics. New York: Academic Press.

Morales, R., Aguiar, A., Subiabre, I. \& Realini, C. E. (2013). Beef acceptability and consumer expectations associated with production systems and marbling. Food Quality and Preference, 29 (2), 166-173.

Noussair, C., Robin, S., \& Ruffieux, B. (2004). Revealing consumers' willingness-to-pay: A comparison of the BDM mechanism and the Vickrey auction. Journal of Economic Psychology, 725-741.

Poole, N., Martínez-Carrasco, L., \& Giménez, F. (2006). Quality perceptions under envolving information conditions: Implications for diet, health and consumer satisfaction. Food Policy, 175-188.

Realini C.E., Kallas, Z., Pérez-Juan, M., Gómez, I., Olleta, J.L., Berian, M.J.; Albertí, P. \& Sañudo, C. (2014) Relative importance of cues underlying Spanish consumers' beef choice 
and segmentation, and consumer liking of beef enriched with n-3 and CLA fatty acids. Food Quality and Preference, 33, 74-85.

Resurreccion, A. (2004). Sensory aspects of consumer choices for meat and meat products. Meat Science, 66(1), 11-20.

Siró, I., Kápolna, E., Kápolna, B. \& Lugasi, A. (2008) Functional food. Product development, marketing and consumer acceptance-A review, Appetite, 51(3), 456-467.

Thurstone, L. (1927) A law of comparative judgement. Psychological Review, 34, 273-286.

Urala, N. \& Lahteenmaki,L. (2003). Reasons behind consumers functional food choices. Nutrition and Food Science, 33, 148-158.

Urala, N., \& Lahteenmaki, L. (2004). Attitudes behind consumer's willingness to use functional foods. Food Quality and Preference, 15, 793803.

Verbeke, W. (2005). Consumer acceptance of functional foods: Sociodemographic, cognitive and attitudinal determinants. Food Quality and Preference, 16, 45-57.

Verbeke, W. (2006). Functional foods: Consumer willingness to compromise on taste for health? Food Quality and Preference, (17), 126-131.

Verbeke, W., Pérez-Cueto, F. J., Barcellos, M. D. D., Krystallis, A. \& Grunert, K. G. (2010). European citizen and consumer attitudes and preferences regarding beef and pork. Meat Science, 84(2), 284-292. 


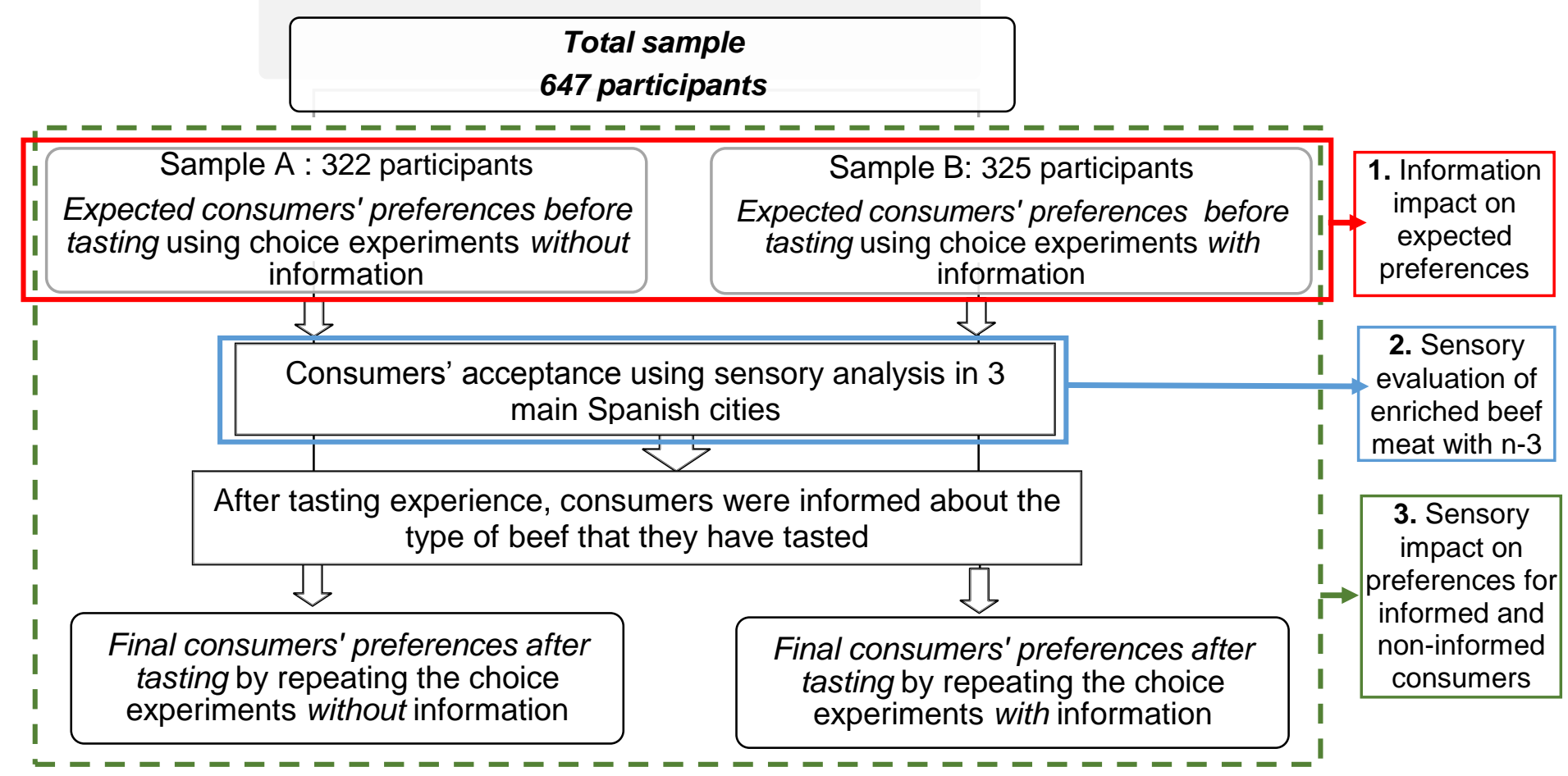

Figure 1: Scheme of the methodological framework 
Table 1: Relative importance of beef attributes (\%) from the G-MNL model

\begin{tabular}{|c|c|c|c|c|}
\hline \multirow{3}{*}{$\beta$} & \multicolumn{4}{|c|}{ Generalized Multinomial Logit model } \\
\hline & \multicolumn{2}{|c|}{ Without information } & \multicolumn{2}{|c|}{ With information } \\
\hline & Pre Sensory & Post sensory & Pre Sensory & Post sensory \\
\hline Fat content & $\begin{array}{l}36.74^{\star \star \star a} \\
(25.1 ; 48.4)\end{array}$ & $\begin{array}{c}19.46 \%{ }^{* \star \star} b \\
(12.2 ; 26.7)\end{array}$ & $\begin{array}{l}19.62^{\star * b} \\
(7.7 ; 31.6)\end{array}$ & $\begin{array}{c}16.45 \%{ }^{* \star *} \mathrm{a} \\
(9.4 ; 23.5)\end{array}$ \\
\hline Colour & $\begin{array}{l}21.88^{\star \star \star a} \\
(13.1 ; 30.6)\end{array}$ & $\begin{array}{c}11.78 \%{ }^{* \star \star} \mathrm{b} \\
(7.2 ; 16.2)\end{array}$ & $\begin{array}{c}16.92^{\star \star \star a} \\
(10.3 ; 23.6)\end{array}$ & $\begin{array}{c}10.57 \%^{* \star \star b} \\
(6.5 ; 14.6)\end{array}$ \\
\hline Origin & $\begin{array}{l}19.34^{\star \star \star a} \\
(12.7 ; 25.9)\end{array}$ & $\begin{array}{c}7.14 \%{ }^{* * * b} \\
(3.2 ; 11.0)\end{array}$ & $\begin{array}{l}15.07^{\star \star \star} a \\
(9.1 ; 21.0)\end{array}$ & $\begin{array}{c}8.62 \%{ }^{* * b} \\
(4.8 ; 12.4)\end{array}$ \\
\hline Diet & $\begin{array}{c}1.72^{b} \\
(-7.6 ; 11.0)\end{array}$ & $\begin{array}{c}35.98 \%{ }^{* \star \star} \mathrm{a} \\
(26.9 ; 45.1)\end{array}$ & $\begin{array}{l}22.73^{\star * \star b} \\
(15.4 ; 30.0)\end{array}$ & $\begin{array}{c}34.79 \%{ }^{* * \star a} \\
(25.8 ; 43.8)\end{array}$ \\
\hline Price & $\begin{array}{l}20.33^{* \star *} a \\
(11.1 ; 29.5)\end{array}$ & $\begin{array}{c}25.66 \%{ }^{* * * a} \\
(19.3 ; 31.9)\end{array}$ & $\begin{array}{l}25.67^{\star \star \star a} a \\
(17.4 ; 33.9)\end{array}$ & $\begin{array}{c}29.57 \%{ }^{* * * a} \\
(23.4 ; 35.8)\end{array}$ \\
\hline
\end{tabular}

Significance levels: ${ }^{* * *} p<0.01 ;{ }^{* *} p<0.05 ;{ }^{*} p<0.10$

a,b: Differences between preferences (pre sensory and post sensory) within each group at $95 \%$. 
Table 2: Results from model estimations for consumer data with and without information

The Generalized Multinomial Logit model

\begin{tabular}{ccc}
\hline Without information & With information \\
Pre sensory Post sensory & Pre sensory Post sensory
\end{tabular}

Random Parameters in utility functions $(\beta)$

\begin{tabular}{|c|c|c|c|c|}
\hline Moderate visible fat & $-0.50^{\star \star \star}$ & $-0.41^{* \star *}$ & $-0.31^{* \star *}$ & $-0.35^{\star \star \star}$ \\
\hline Pale red & $-0.30^{* * *}$ & $-0.25^{\star * *}$ & $-0.27^{* * *}$ & $-0.22^{\star * \star}$ \\
\hline Other Spanish origin & $-0.26^{\star \star}$ & $-0.15^{\star \star}$ & $-0.24^{* \star \star}$ & $-0.18^{\star \star \star}$ \\
\hline Enriched with $n-3$ & 0.03 & $0.58^{\star * \star}$ & $0.19^{* * *}$ & $0.42^{\star \star \star}$ \\
\hline Enriched with CLA & -0.01 & 0.12 & 0.01 & 0.10 \\
\hline Enriched with n-3 \& CLA & 0.11 & $0.22^{*}$ & $0.33^{\star * \star}$ & $0.49^{* * *}$ \\
\hline Price $6.6 €$ (high) & $-0.52^{\star \star \star}$ & $-0.69^{* \star *}$ & $-0.67^{* * *}$ & $-0.87^{\star * \star}$ \\
\hline Price $5.7 €$ (medium-high) & $0.11^{\star \star \star}$ & -0.07 & $0.20^{* \star *}$ & 0.02 \\
\hline Price $4.8 €$ (medium-low) & $0.37^{* * *}$ & $0.38^{\star * *}$ & $0.32^{\star * *}$ & $0.41^{* * *}$ \\
\hline Opt-Out & $0.48^{* \star \star}$ & $0.44^{* \star *}$ & -0.15 & 0.16 \\
\hline
\end{tabular}

Independent standard deviations of parameters distribution obtained from Diagonal values in Cholesky matrix

\begin{tabular}{|c|c|c|c|c|}
\hline & & & & \\
\hline Moderate visible fat & $1.63^{\star \star \star}$ & $1.58^{\star \star \star}$ & $2.14^{\star \star \star}$ & $1.82^{\star \star \star}$ \\
\hline Pale red & $0.94^{* * *}$ & $0.10^{* *}$ & $0.89^{* \star \star}$ & $0.11^{*}$ \\
\hline Other Spanish origin & $0.12^{*}$ & 0.08 & $0.16^{\star * *}$ & $0.26^{\star * *}$ \\
\hline Enriched with $n-3$ & $0.30^{\star \star \star}$ & 0.05 & 0.02 & $1.33^{* * *}$ \\
\hline Enriched with CLA & 0.02 & $1.06^{\star \star \star}$ & 0.01 & $0.28^{* *}$ \\
\hline Enriched with n-3 \& CLA & $0.21^{* \star}$ & $0.81^{* \star *}$ & 0.15 & $0.25^{\star *}$ \\
\hline Price $6.6 €$ (high) & $0.30^{* *}$ & $0.90^{* * *}$ & 0.17 & $1.48^{* \star \star}$ \\
\hline Price 5.7€ (medium-high) & $.42^{* \star \star}$ & $0.24^{* *}$ & $0.25^{* *}$ & 0.20 \\
\hline Price $4.8 €$ (medium-low) & 0.06 & $0.14^{* *}$ & $0.28^{* *}$ & 0.03 \\
\hline Opt-Out & $1.27^{\star \star \star}$ & $0.42^{\star \star \star}$ & $1.65^{\star \star \star}$ & $1.71^{* * *}$ \\
\hline \multirow{3}{*}{$\begin{array}{r}\text { Variance parameter in scale parameter } \tau \\
\text { Weighting parameter } \gamma\end{array}$} & \multicolumn{4}{|c|}{ scale parameters } \\
\hline & $0.56^{\star \star \star *}$ & \multirow{2}{*}{$\begin{array}{c}0.08 \\
0.31^{\star * *}\end{array}$} & \multirow{2}{*}{$\begin{array}{l}0.12^{*} \\
0.10\end{array}$} & 0.11 \\
\hline & $0.34^{\star \star \star}$ & & & 0.10 \\
\hline Log-Likelihood $(\theta)$ & $-2,658.67$ & -2705.29 & -2656.7667 & -2801.51 \\
\hline Log-Likelihood (0) & $-3,571.09$ & $-3,571.09$ & $-3,604.36$ & $-3,604.36$ \\
\hline LL ratio test & $\begin{array}{c}1,824.84 \\
(0.000)\end{array}$ & $\begin{array}{c}1,731.60 \\
(0.000)\end{array}$ & $\begin{array}{c}1,895.19 \\
(0.000)\end{array}$ & $\begin{array}{c}1605.69 \\
(0.000)\end{array}$ \\
\hline Pseudo $\mathrm{R}^{2}$ & 0.255 & 0.242 & 0.262 & 0.227 \\
\hline $\mathrm{AIC} / \mathrm{N}$ & 2.124 & 2.160 & 2.103 & 2.214 \\
\hline
\end{tabular}

Significance levels: ${ }^{* * *} p<0.01 ;{ }^{* *} p<0.05 ;{ }^{*} p<0.10$ 
Table 3. Overall acceptability scores of beef from animals fed different diets assigned by consumers.

\begin{tabular}{|c|c|c|c|c|}
\hline \multirow[b]{2}{*}{ Type of beef meat } & \multicolumn{2}{|c|}{ Overall acceptability } & \multirow[b]{2}{*}{$P$ value ${ }^{*}$} & \multirow{2}{*}{$\begin{array}{l}\text { Whole } \\
\text { Sample }\end{array}$} \\
\hline & $\begin{array}{c}\text { Without } \\
\text { information }\end{array}$ & $\begin{array}{c}\text { With } \\
\text { information }\end{array}$ & & \\
\hline Conventional & 5.73 & 5.70 & 0.858 & $5.71^{c}$ \\
\hline Enriched with omega-3 & 6.17 & 6.10 & 0.611 & $6.14^{\mathrm{a}}$ \\
\hline Enriched with CLA & 6.04 & 5.76 & 0.051 & $5.90^{b, d}$ \\
\hline Enriched with omega-3 \& CLA & 5.74 & 5.79 & 0.712 & $5.76^{c, d}$ \\
\hline
\end{tabular}

Differences between mean scores assigned by consumers with and without information. $a, b, c, d$ Statistical differences among types of beef meat for all consumers at $95 \%$. 